\title{
AUTOMATION FRAMEWORK-STRATEGY TO QUALITY SOFTWARE
}

\author{
Janisha Jayan ${ }^{1}$, Apoorva Manikumar.V ${ }^{2}$, Bhuvana.G.M ${ }^{3}$, Roopashree $\mathbf{S}^{\mathbf{4}}$ \\ ${ }^{I}$ Final year B.E. student, Department of Computer Science, TJohn Institute of Technology, Karnataka, India \\ ${ }^{2}$ Final year B.E. student, Department of Computer Science, TJohn Institute of Technology, Karnataka, India \\ ${ }^{3}$ Final year B.E. student, Department of Computer Science, TJohn Institute of Technology, Karnataka, India \\ ${ }^{4}$ Assistant Professor, Department of Computer Science, TJohn Institute of Technology, Karnataka, India
}

\begin{abstract}
"Automation framework" is created using Java and selenium Technology. As this is developed in Java, ittherefore incorporates all features of the Java Technology. The main purpose of this project would be to check the quality of any web application before the release. This frame work will help to perform black box testing to ensure quality software is developed and delivered to the customer. This frame will be platform independent. It will work on Windows OS, MAC, and Linux etc. And the end of test execution the software will generate HTML based report for better analysis of failures. For end user to simply there testing I will develop .bat file, so that just on one click project execution will start. This frame work will help to perform black box testing to ensure a quality software is developed and delivered to the customer. This frame will be platform independent. It will work on Windows OS, MAC, and Linux etc. And the end of test execution the software will generate HTML based report for better analysis of failures. For end user to simply there testing I will develop .bat file, so that just on one click project execution will start. The main objective, is always to decrease time and the cost software testing requires, while assuring and maintaining application quality. Automation tools allows organizations to run tests repeatedly in a swift manner. The tools manage test execution, report outcomes and compare results with earlier test runs.A key advantage of test automation is the ability to easily repeat tests
\end{abstract}

Keywords: Automation, Testing Tool, Framework, Selenium, Quality of Software.

****

\section{INTRODUCTION}

Automated testing tools are used for executing tests, reporting outcomes and comparing results with earlier test runs. Tests carried out with these tools can be run repeatedly, at any time of day. Scope of automation is the area of the Application Under Test which will be automated. Following help determine scope:

- Business critical features.

- Scenarios with a huge amount of data

- Applications with similar functionalities.

- Technical usability.

- Reusability of business components are extended.

- Test cases complexity.

- Reusability of same test cases for cross browser testing.

The main objective of this software is to provide agile way of testing and reduce regression efforts.It also performs Function/Integration testing,System testing and smoke testing.It also reduces recruitment cost by replacing manual efforts with automation. Automation can be used for testing Login forms, Registration forms, GUI Items, field validations, database connections.

\subsection{Advantages}

- Reliable: It helps in eliminating human error, as the same operations are carried out.

- Reusable: Tests can be reused on different versions of an application anywhere and anytime.
- Better Quality Software: It uses fewer resources to run more tests in very less time.

- Speed: It runs tests faster than human users and hence there is a considerable increase in speed .

- Cost Reduction: The number of resources used for tests are reduced and hence requires very less initial and total investments .

- Replaces: It replaces manually mundane and labor intensive tasks and works better and in an easier manner.

\subsection{Disadvantages}

- Initial and total investment is very high for tools and training.

- Manual efforts are required for test preparation.

- Testing areas are mostly left uncovered.

- Automated testing does not replace manual testing but is an addition to it.

The usual manual testing process consists of the following:

1. Functional Testing: It is also known as unit testing.It is the process in which every component or features of an application is tested thoroughly or vigorously.It checks the functionality of each component.This is the first step in testing and the testing process begins with this.The automation framework helps in skipping this step by step process and hence making the process easier and faster. There involves no delay and is user friendly. 
Eg: testing of all the features such as the username, password etc.

2. Integration Testing: It is the process of testing the data flow between two features of an application. It ensures that the data flow starting from one feature reaches the intended feature in a proper manner. It sees to it that the communication between two features of the same or different applications is proper .

Eg: Sending and receiving emails in Google.

3. System Testing: It is also known as end-to-end testing. It performs the complete testing of the application from one end to another end.

The Automation Framework performs the whole testing process in one step. This is a one step solution to testing. It contradicts with the normal testing process which actually takes a lot of time to complete. This completes testing any web application in a maximum of 10 minutes. This property has found to be advantageous for a normal tester who has to spend a long time and put a lot of his efforts.

\section{WORKING}

The main objective is to develop a web application called the "Automation Framework" that is used to make the process of black box testing easier.The project is run using the waterfall model. The Waterfall model is a most important model of the Software Development Life Cycle model for Software Engineering. SDLC is the step by step procedure of developing a software.It is a traditional model It is linear and sequential approach to develop and check the quality of the software. After a phase of development is done, the development leads to the next phase and there it won't go back since the reverse process is not possible. The most important benefit of waterfall development model is that it allows to categorize into departments and managerial control. Steps involved in Automation Process are

1. Locate fields using xpaths by building properties file.

2. Testing process is carried out by JUnit.

3. Automation,Compilation,Execution and Generation of HTML reports can be done by ANT.

4. bat files are generated.

5. Connection to database is achieved using JDBC.

Automation testing supports the following:

- Regression testing can be performed frequently.

- Feedback to Developers are rapid and instant and hence no delay.

- The number of test case executions are unlimited.

- Agile and extreme development methodologies are greatly supported.

- Test cases are documented in a disciplined manner.

- Precise and accurate defect reporting.

- Defects missed when performing manual testing are easily detected.
Automation testing controls the execution of tests using a special software and compares the actual results with the predicted results.

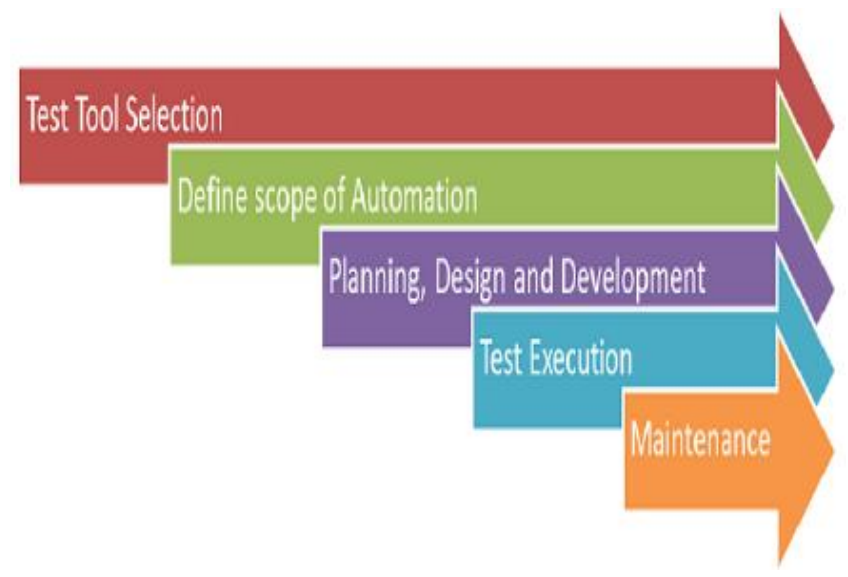

Fig -1: Steps in Automation Process

Automation reduces efforts and cost requirements. It is quick and allows test engineers to focus on functional areas. It provides complete reusability based on the type of implementation. It can be used for any tests including load, sanity, system etc. The script validates all areas once tested. It improves accuracy and ensures consistency. It provides wider test coverage of application features and the results are reliable.These tools helps to carry out tests which can be run repeatedly, any number of times.

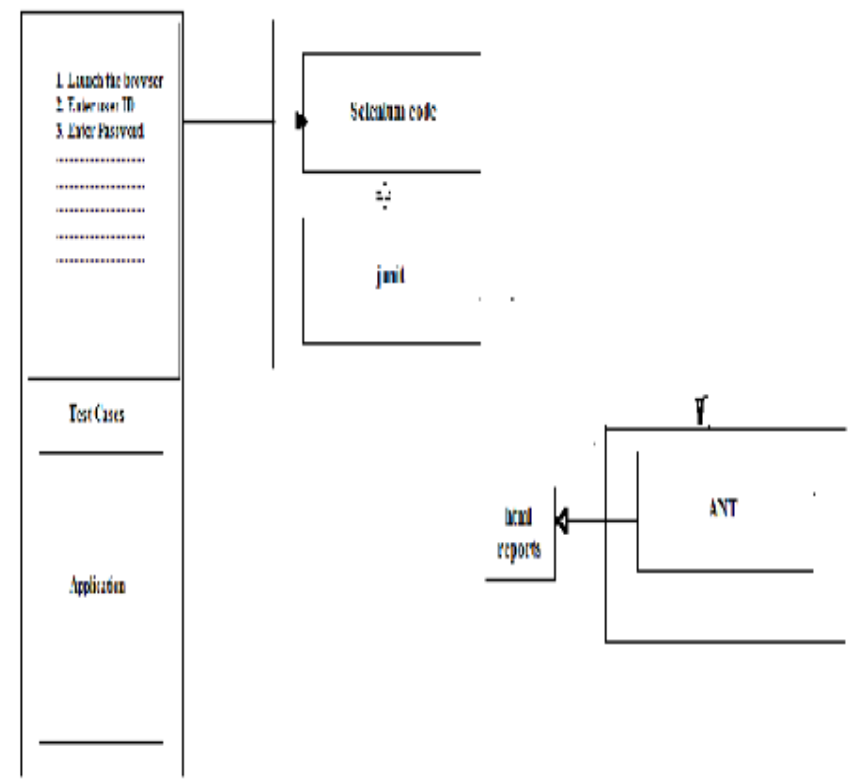

Fig -2: System architecture of Automation Framework

\subsection{Procedure}

\subsubsection{Generating Test Cases}

User should create test cases in Microsoft Excel. 


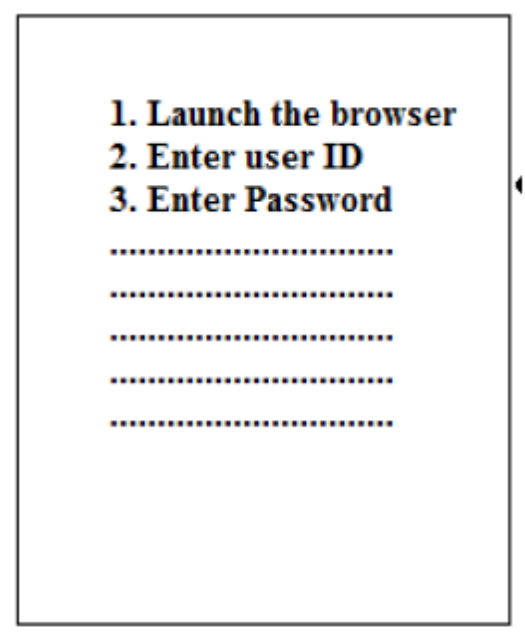

\section{Excel File}

Fig-3: Test cases in Microsoft Excel

These test cases along with the application are given as input to the Selenium code.

\subsubsection{Testing}

The test cases and the application are taken as input to the selenium code.

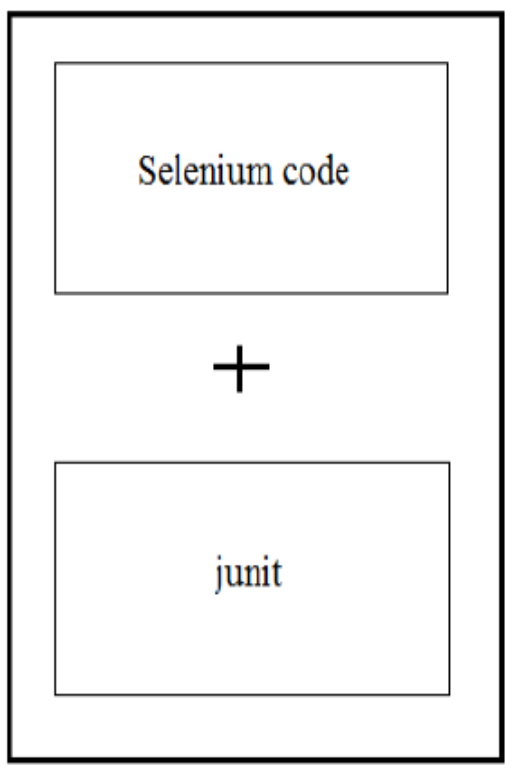

Fig-4: Testing for finding defects

The given input is then tested using Selenium code and JUnit. JUnit in Java is a framework for unit testing. It has mainly been used for developing the test-driven development. It is one among the category of unit testing frameworks which is together called as xUnit, which came up with the Junit.

\subsubsection{Defect Tracking}

The defects found from testing using the selenium code and JUnit are reported to ANT using Bugzilla defect tracking system.

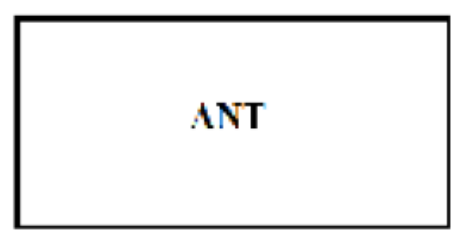

hrmal soport

Fig-5: Defect Tracking

ANT then uses this information to generate html reports about the defects. The defects include any errors that cause the application to not run properly or that affects the quality of the application.

In Automation Framework ,the user has to first create test case in Microsoft Excel. The user is free to generate any number of test cases for the same application. This helps in making the process easier.

These test cases along with the application is given as input to the unit which includes the Selenium code and JUnit. Selenium is a collection of jar files. Jar file is a collection of .class files. The .class files are generated after compiling the java program. It consists the binary form of the java program. Since Selenium cannot test all the functions and all applications and hence seeks the help of JUnit that helps in unit testing.

The Selenium code once after receiving the application and test cases from the excel file as input, will then use it to test to find defects in the respective application. Hence the defects that affects the quality of the software is found before its release.

These defects are tracked using the BUGZILLA defect tracking system. These defects are then given as inputs to the ANT .Software building process is automated by ANT. It is an open source tool. It is a build tool which is based on JAVA. It helps a developer and tester in their day-to-day schedule. The development code is built to deployment utilities. Applications are built using ANT libraries. It can create documents in Java.

All the process executes as a .bat file. The defects after giving as input to the ANT, it will then generate html reports on the defects found. This comes out as results and helps the tester to note the defects and report it to the developer, who will then take necessary steps to rectify the error or defects and produce a quality software that meet the requirements and necessities of the customer.

Hence,an Automation Framework eases the work of the tester tremendously and with a very high accuracy. The accuracy of testing is very high using the Automation Framework .It is the most cost effective method of testing and requires less number of resources with little or no delay. 


\subsection{Applications of Automation Testing}

- It uses a generic framework and can be used to test any web application designed in any language such as Java, PHP etc. Eg :Gmail ,Quiker ,Flipkart ,CRM,ERP etc.

- It is used to avoid human errors.

- It is used for Mission Critical Business and for long term maintenance of software.

- It is used to check the real time performance of the application.

\section{CONCLUSION}

Automation Testing is the process of using a software to control the test execution. It involves automating a manual process already in place that uses a formalized testing process. It reduces impact at the bottom line of regression testing and also increases the flexibility in time and resources and also increases the speed and precision. Automated testing tools have the ability of executing tests, reporting outcomes and comparing results with earlier test runs. Better and quality software is the result of running the right tests and continually re-evaluating the right tests to be executed.

\section{REFERENCES}

[1]. https://www.hcltech.com/sites

[2]. https://www.infosys.com/IT-services

[3]. www.gallop.net/whitepaper-scriptless-test-automation

[4]. www.agreeya.com/WhitePaper/TestAutomation

[5]. "End-to-end test automation - A behavior-driven and tool-agnostic approach"- Anand Avinash Tambey,2009

[6]. "Testing Automation Framework Using MBT"Rober.V.Binder,2008

[7]. "Developing a successful Point-of-Sale (POS) test automation strategy"-Sujith George, 2009

[8]. "Test Automation and Software Development, Janardhana.S

[9]. "Strategy for Testing Merger of Banks"-Puneeth Shukla,2011

[10]. "Enhance Mobile Application Performance with Successful Network Impact Testing"-Manik Jandial,2011

\section{BIOGRAPHIES}

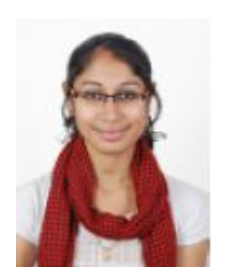

Janisha Jayan, Eighth Semester, Department of Computer Science and Engineering, $\mathrm{T}$ John Institute of Technology

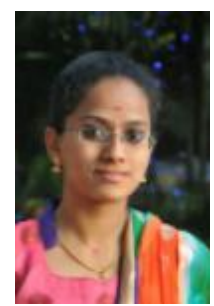

Apoorva Manikumar.V, Eighth Semester, Department of Computer Science and Engineering, $\mathrm{T}$ John Institute of Technology

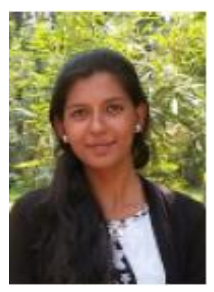

Bhuvana.G.M, Eighth Semester, Department of Computer Science and Engineering, $\mathrm{T}$ John Institute of Technology

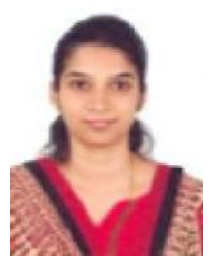

Prof. Roopashree $\mathbf{S}$ received her $\mathrm{BE}$ degree in 2001 from Department of Computer Science and Engineering, VTU and MTech in Computer Science and Engineering, VTU. She is currently pursuing her $\mathrm{PhD}$ in Department of Computer Science and Engineering, VTU in Machine Learning and Artificial Intelligence. She has published various papers in National and International journals. 